\title{
Long-Term Chemical Resistance of Ecological Epoxy Polymer Composites
}

\author{
Bernardeta Dębska', Lech Lichołai' \\ 1 Department of Building Engineering, Rzeszow University of Technology, Poznańska 2 St., 35-959 Rzeszów, \\ Poland \\ * Coresponding author's e-mail: bdebska@prz.edu.pl
}

\begin{abstract}
Resin concretes belong to a small group of building materials which, besides high strength parameters, also have a very good chemical resistance. This is confirmed by the studies carried out by various research institutions around the world. However, there is little data on the behaviour of composite resin exposed to corrosive solutions for an extended period of time. This article presents the results of the research on weight changes in samples of epoxy mortar modified with poly(ethylene terephthalate) glycolysates, immersed for 5 years in four different aggressive media i.e. 10\% aqueous solutions of sulphuric and nitric acids, sodium hydroxide, and sodium chloride. The actual average weight changes obtained were compared with the data calculated on the basis of the regression functions fitted to the data recorded after 3.5 years of exposure. This allowed verification of the model selection correctness and evaluation of the effectiveness of the fitted regression curve. In the case of aqueous sodium hydroxide and sodium chloride solutions, it can be assumed that the logarithmic model describes weight changes well. It was observed that the weight of the samples exposed to $\mathrm{NaCl}$ solutions and $\mathrm{NaOH}$ stabilizes over prolonged monitoring time and reaches a plateau. However, the weight changes in mortar samples immersed for 5 years in aqueous solution of sulphuric and nitric acids quite significantly differ from the data calculated on the basis of the trend line fitted to the results of the tests carried out after 3.5 years of exposure. It seems that the better solution in this case is the selection of an exponential model. In addition, placing the logarithmic trendlines for all corrosive media together on a chart allows to note which of the solutions is the most aggressive. It was found that after 5 years of immersion in aqueous solutions of acids, mortar samples became brittle, and the observation of their fractures confirmed the weakness of the connection on the resin/aggregate phase boundary. Changes in the appearance of the samples were also noted, namely the surface of samples submerged in a solution of nitric acid strongly yellowed, and those treated with sulphuric acid were tarnished.
\end{abstract}

Keywords: modified epoxy mortars, long-therm resistance, chemical resistance prognostic models, PET waste

\section{INTRODUCTION}

Chemical resistance is a key feature of, inter alia, the cases where concrete buildings are constructed near marine waters, road and bridge drainage systems and sewage systems, tanks for containing aggressive substances, industrial tanks and wells, industrial floors (Gizelter 2014). A number of traditional building materials are not suitable for the applications where they are exposed to corrosive substances. One solution to the limitations associated with the high risk of chemical aggression may be resin concrete. This material is formed by mixing aggregates, synthetic resin and an appropriately selected hardener. The most commonly used resins include the thermosetting resins i.e. polyester, epoxy and acrylic [Czarnecki 2010, Lokuge and Aravinthan 2013, Dębska and Lichołai 2016]. These binders show good chemical resistance in aggressive environments, as well as concretes and mortar prepared on the basis of these polymers tend to reproduce the characteristics of the binders used.

Scientists around the world are conducting research on the chemical resistance of polymer composites, including resin and resin mortars [Rahimi et al. 2016, Stamenović et al. 2011, Mortas et al. 2014, Yan et al. 2015, Amaro et al. 2013, 
Feng et al. 2014, Garcia-Espinel et al. 2015, Suihkonen et al. 2016, Lu et al. 2016, Gorninski et al. 2007, Reis 2010].

Reis [2009] immersed the samples of cement and polyester mortars in a $10 \%$ solution of hydrochloric acid and 10\% solution of sulphuric acid for a period of 28 days. For samples of polyester mortars, he found no loss of weight, while the weight of the cement-based mortar samples in the same experiment fell by c. $50 \%$ of the initial weight. A very good resistance to $10 \%$ solutions: sulphuric acid, sodium hydroxide and sodium chloride, in epoxy mortar samples was also found by the team of Md. M. Rahman [2015]. After 12 months of exposure, the weight changes and changes in compressive strength did not exceed $1 \%$ and depended on the amount of resin in the composite.

R. Griffiths and A. Ball [2000] determined the strength of glass fibre reinforced polyester concrete, inter alia, after 3 weeks of exposure to $10 \%$ aqueous solutions of sulphuric acid, hydrochloric acid and sodium hydroxide. A 15-day immersion period applied in the abovementioned aqueous solutions resulted in the loss of original strength, at the level of $18 \%, 24 \%$ and $35 \%$, respectively. In turn, seven-month immersion of samples in $\mathrm{NaCl}$ solution resulted in a reduction in their strength by $35 \%$.

The beneficial effect on chemical resistance of resin composites can be attributed to microfillers, which in a certain way reduce the porosity of the resin concrete, and thus limit the penetration of the solutions to the interior of the composites [Ghorbel and Haidar 2016]. The authors compared the resistance of epoxy and cement concretes immersed in solutions of sulphuric, hydrochloric and citric acids for a period of 6 months. They produced two types of epoxy concrete, containing $9 \%$ and $7 \%$ epoxy resin by weight. The reduction of resin quantity was achieved through the introduction of calcareous fillers. The concretes obtained were resistant to the action of citric and sulphuric acids. Small weight changes were observed in the samples. In turn, the team of G. Mehdi produced the samples of epoxy concrete containing two different microfillers - fly ash and silica powder [Golestaneh et al. 2013]. These composites were subjected to the action of $15 \%, 30 \%$ and $60 \%$ solutions of sodium hydroxide, hydrochloric acid, sulphuric, citric and acetic acids. The authors evaluated the physical properties (appearance of samples and test medium) and chemical resistance (compressive strength and weight changes) in the concrete samples produced after 7, 14, 28 and 56 days of immersion. The epoxy concrete obtained was characterized by high chemical resistance. The samples immersed in a solution of acetic acid were an exception. The weight loss was in this case c. $31 \%$. There was also a significant decrease in the compressive strength of the samples immersed in $60 \%$ aqueous solutions of sulphuric and acetic acids.

The positive impact of adding micro-filler in the form of fly ash on the chemical resistance of concrete with a matrix created from polyester resin or vinyl ester was also confirmed in the study carried out by A. Garbacz and J.J. Sokołowska [2013]. After 48 hours of immersion in aqueous solutions of sulphuric acid and potassium hydroxide, the majority of samples were characterized by changes at the level of no more than $0.5 \%$. Ribeiro et al. [2002] determined a weight change and a change in the flexural strength in polyester and epoxy concretes immersed in water and 10\% aqueous solutions of sulphuric acid and sodium chloride. After 84 days of exposure, the weight changes in polyester concrete samples did not exceed $0.45 \%$ for water, $0.25 \%$ for sulphuric acid and $0.15 \%$ for $\mathrm{NaCl}$. For comparison, the wright changes of epoxy concrete immersed in the same media were $0.09 \%, 0.1 \%$ and $0.21 \%$. The changes in the flexural strength of polyester concrete immersed in sulphuric acid amounted to even $30 \%$. In the case of the epoxy concrete, this change was three times lower. The authors pointed out the need to carry out research over longer periods of time to model the dependencies obtained.

Despite the long-standing application of polymer composites as important building materials, it is difficult to find the results of studies that model the changes to their properties caused by the prolonged action of aggressive media. Usually, the authors of such publications are unanimous in finding that the chemical degradation mechanisms adversely affect the connection between the polymer matrix and aggregate [Rahman et al. 2015, Griffiths and Ball 2000]. The diffusion of liquid solutions through the material can be fast, especially in the cases where there is a high content of empty spaces in the structure of the composites. A reduction in bonding forces on the phase boundary leads to a decrease in the stress parameters. Solutions of acids and bases can attack the material from the interfacial positions and degrade the polymer matrix itself. Taking into account the 
physical mechanism of this phenomenon, the increase in weight of samples resulting from the absorption of the solution through the sample seems to be a process that stabilizes over the longer period of time. Choosing the right model for predicting the properties of resin composites exposed to corrosive solutions, however, is not easy to do, especially in long-term forecasts, which require data collection during prolonged periods to monitor the changes to properties.

This article describes the results of studies of the long-term impact of corrosive solutions on the changes in weight and appearance of epoxy mortar modified with poly(ethylene terephthalate) (PET) glycolysates. The modification consisted in the partial substitution of epoxy resin mortars by poly(ethylene terephthalate) glycolysates. The poly(ethylene terephthalate) was from, inter alia, waste soft drinks bottles, which were cleaned and shredded, and then underwent the process of glycolysis using the appropriate glycol. With regard to the study of chemical resistance, it was assumed that the types of corrosive environments that may have potential contact with products in reality will be simulated, including: $10 \%$ solution of sulphuric acid, $10 \%$ solution of nitric acid, $10 \%$ solution of sodium hydroxide and $10 \%$ solution of sodium chloride. The process of monitoring the changes to the composite properties lasted 5 years, and studies were carried out in the period 2011-2016. The trend lines were adjusted to the data obtained after 3.5 years of immersion of samples in various aqueous solutions by choosing two different models for this purpose: logarithmic and exponential. Then, by means of the extrapolation method, the projected changes in values of samples after 5 years of exposure were calculated and compared with the actual data found experimentally after five years of immersion of samples in the abovementioned aqueous solutions of aggressive media.

\section{EXPERIMENTAL PROTOCOLS}

\section{Materials}

Epoxy resin Epidian 5 (produced by Organika Sarzyna S.A.) was applied to obtain the resin mortar. For hardening, resin hardener Z-1 (triethylenetetraamine) was used in the amount of $10 \% \mathrm{w} / \mathrm{w}$ in relation to the resin. Quartz of sand grain size $0-2 \mathrm{~mm}$ constituted the aggregate, in accordance with the requirements of PN EN 196-1. On the basis of the available literature data and our own preliminary research results concerning this type of mortar, it was established that the range of the ratios of resin to aggregate will be from 0.14 to 0.36 , in accordance with the adopted experiment plan. In the mortars obtained epoxy resin was partially replaced ( $0-14 \%$ by weight) by poly(ethylene terephthalate) glycolysates. A detailed description pertaining to the selection of the plan is described in [Lichołai and Dębska 2014]. In the case of the research on chemical resistance, the plan took the form of a table (Table 1).

Each of the ten columns of the table presents one point of the plan and describes the composition of the mortars examined. The plan that was selected allowed for a repeat of the research at the central point; that is why the plan points 5 and 6 do not differ in composition. The results obtained during the experiment were analysed by means of the STATISTICA software.

Four different types of waste PET glycolysates based on glycols were used to prepare the sample: propylene (P3), diethylene (P4), dipropylene (S4) and butylene (S5).

Appropriate amounts of epoxy resin and the modifier were weighed and mixed for uniformity. Then, the components were heated up to $85^{\circ} \mathrm{C}$ for 60 minutes to trigger the reaction of the functional groups of the two components. When the epoxy mixture modified with PET glycolyzate reached the room temperature, it was carefully mixed with an appropriate amount of Z-1 hardener (10 parts of water/100g of resin) until a uniform mixture was obtained. The previously prepared resin mixtures were put into the bowl of the laboratory mixer and mixed with standardized sand maintaining the same mixing time and the rotational speed of the mixer. The mortar obtained was put into $60 \times 60 \times 5 \mathrm{~mm}$ metal moulds. The specimens were left for curing in the laboratory atmosphere for seven days.

Table 1. Lists of parameters describing mortar composition for particular points of the plan

\begin{tabular}{|l|c|c|c|c|c|c|c|c|c|c|}
\hline \multicolumn{1}{|c|}{ No of experiment plan point } & 1 & 2 & 3 & 4 & 5 & 6 & 7 & 8 & 9 & 10 \\
\hline Content of PET glycolyzate, \% by weight & 7.03 & 2.06 & 0.00 & 7.03 & 7.03 & 7.03 & 12.00 & 12.00 & 14.06 & 2.06 \\
\hline Resin-aggregate mass ratio Z/K & 0.36 & 0.33 & 0.25 & 0.14 & 0.25 & 0.25 & 0.18 & 0.33 & 0.25 & 0.18 \\
\hline
\end{tabular}




\section{Methods}

The chemical resistance test was performed according to PN-EN ISO 175:2002, at a temperature of $23 \pm 2^{\circ} \mathrm{C}$. After being dried to a solid at $50 \pm 2^{\circ} \mathrm{C}$, the weight of each specimen was recorded at an accuracy of $1 \mathrm{mg}$. Then, the specimens were put in containers with the particular corrosive solutions. They were hung in such a way that the corrosive agent had an easy access to all the surfaces of the specimens. Each type of mortar (modified with different glycolyzate) was put in a separate container. After a fixed time of immersion in the aggressive substance (1-7 days, 2 weeks, 4 weeks, 16 weeks, 6 months, 12 months, 3.5 years and 5 years, respectively) the specimens were taken out, dried and then weighed. After denotation they were put back into the containers with the corrosive substance and kept there until the next weighing.

For each piece the percentage change was calculated $(\Delta \mathrm{m})$ using the equation:

$$
\Delta m=\frac{m_{2}-m_{1}}{m_{1}} \times 100 \%
$$

where: $m_{1}-$ is the weight of the sample expressed in milligrams (mg), after the initial drying before immersion in the aggressive medium,

$m_{2}-$ is the weight of the sample expressed in milligrams ( $\mathrm{mg}$ ), after a specified duration of immersion in the selected aggressive medium.

The final result is expressed as the arithmetic mean of the three weight values obtained for three samples, after the same duration time of immersion. The STATISTICA software was used for the statistical analysis of the experiment results. In addition, the changing colour of samples was visually evaluated.

\section{RESULTS AND DISCUSSIONS}

The models of the regression function that describes a specific phenomenon present very valuable information in a particular field of science. The modern calculation methods and statistical tools enable to select functions with many variables which fit well with the data analysed. The choice of the model should not only be based on the knowledge of the rules resulting from the sta- tistical calculation results (e.g., due to a high value of correlation coefficients or determinations), but also on the basis of knowledge of the phenomenon under study [Czarnecki and Sokołowska 2015]. In the case of the studies modelling changes in the weight of epoxy mortar samples exposed to corrosive solutions, two different regression curves can match the description of the experimental data: logarithmic and exponential with general patterns taking the appropriate forms (2) and (3):

$$
\begin{gathered}
y=a+b \cdot \ln (x) \\
y=a \cdot x^{b}
\end{gathered}
$$

On the basis of the results pertaining to the weight determination of the tested samples, which were immersed in aggressive solutions for a period of 3.5 years, the coefficients of regression equations were set (Table 2). All of the coefficients occurring in the equations were significant. Tables 3 and 4 show the projected and actual average weight changes of mortar samples after five years of immersion in corrosive solutions, extrapolated for the logarithmic and exponential models.

The last column of both tables shows the calculated relative error of the model. On this basis, it can be concluded that in the case of the exposure of epoxy mortar samples to a solution of sodium hydroxide, a much better fit to the actual data was obtained by the application of the regression curve in the logarithmic form. The regression curves designated for mortars modified with P3 glycolysates after 3.5 years of immersion and after 5 years of exposure are very close to each other, as shown in Figure 1.

When other types of glycolysates were used, a similar dependency has been observed. Taking into account the knowledge on the phenomenon under study, it can be expected that the increase in the weight of the sample resulting from the absorption of the solution by the mortar will be a process that asymptotically tends toward fixed values. Thus, the selection of a regression curve in the form of a logarithmic function, according to which growth is much slower in time than in the case of the exponential model, and finally reaches a plateau, seems appropriate. However, in the case of mortar samples immersed in aqueous solutions of acids, the relative errors of the model obtained for both types of regression curves are similar, in particular for the samples immersed in a solution of sulphuric acid (Table 3 and Table 4). 
Table 2. Summary of coefficients of regression equations and coefficients of determination for data obtained after 3.5 years of exposure in aggressive aqueous solutions

\begin{tabular}{|c|c|c|c|c|c|c|c|}
\hline \multirow{3}{*}{$\begin{array}{l}\text { Type of corrosive } \\
\text { solution }\end{array}$} & \multirow{3}{*}{$\begin{array}{c}\text { Type of } \\
\text { glycolysate }\end{array}$} & \multicolumn{6}{|c|}{ Model } \\
\hline & & \multicolumn{3}{|c|}{ Logarithmic, $y=a+b \cdot \ln x$} & \multicolumn{3}{|c|}{ Exponential, $y=a \cdot x^{b}$} \\
\hline & & a & $\mathrm{b}$ & $\mathrm{R}^{2}$ & a & $\mathrm{b}$ & $\mathrm{R}^{2}$ \\
\hline \multirow{4}{*}{$\mathrm{H}_{2} \mathrm{SO}_{4}$} & S4 & -1.13697 & 1.04843 & 0.81109 & 0.22254 & 0.51719 & 0.98868 \\
\hline & S5 & -0.99491 & 0.91364 & 0.76592 & 0.14882 & 0.55995 & 0.98917 \\
\hline & P3 & -0.73388 & 0,9448 & 0.74309 & 0.24196 & 0.50145 & 0.97085 \\
\hline & $\mathrm{P} 4$ & -0.80592 & 0,97242 & 0.79759 & 0.31173 & 0.46049 & 0.96872 \\
\hline \multirow{4}{*}{$\mathrm{HNO}_{3}$} & S4 & -1.50323 & 1,35665 & 0.88149 & 0.45982 & 0.43710 & 0.93149 \\
\hline & S5 & -1.16679 & 1,17618 & 0.89101 & 0.44007 & 0.42698 & 0.95755 \\
\hline & P3 & -1.18657 & 1,16571 & 0.81814 & 0.34271 & 0.4665 & 0.94554 \\
\hline & $\mathrm{P} 4$ & -1.16885 & 1,19513 & 0.84889 & 0.41424 & 0.43965 & 0.94449 \\
\hline \multirow{4}{*}{$\mathrm{NaOH}$} & S4 & 0.11872 & 0,36163 & 0.97781 & 0.47852 & 0.25663 & 0.95088 \\
\hline & S5 & -0.07274 & 0,36073 & 0.97257 & 0.33954 & 0.29692 & 0.94024 \\
\hline & P3 & 0.16934 & 0,35143 & 0.96643 & 0.47425 & 0.26159 & 0.99316 \\
\hline & P4 & 0.33947 & 0,32123 & 0.97212 & 0.58679 & 0.22506 & 0.99004 \\
\hline
\end{tabular}

Table 3. Summary of expected and actual average mortar sample weight changes after 5 years of exposure in aggressive media, according to the logarithmic model

\begin{tabular}{|c|c|c|c|c|}
\hline Type of corrosive solution & Type of glycolysate & $\Delta \mathrm{m}_{\mathrm{p}}, \%$ & $\Delta \mathrm{m}_{\mathrm{e}}, \%$ & $\Delta \mathrm{m}$ \\
\hline \multirow{4}{*}{$\mathrm{H}_{2} \mathrm{SO}_{4}$} & S4 & 6.73604 & 8.85590 & 0.23937 \\
\hline & S5 & 5.86593 & 7.93176 & 0.26045 \\
\hline & P3 & 6.36094 & 8.47583 & 0.24952 \\
\hline & P4 & 6.49631 & 7.96731 & 0.18463 \\
\hline \multirow{4}{*}{$\mathrm{HNO}_{3}$} & S4 & 8.68431 & 9.97358 & 0.12927 \\
\hline & S5 & 7.66554 & 8.82826 & 0.13170 \\
\hline & P3 & 7.56714 & 9.36220 & 0.19173 \\
\hline & P4 & 7.80578 & 9.74393 & 0.19891 \\
\hline \multirow{4}{*}{$\mathrm{NaOH}$} & S4 & 2.83429 & 2.61884 & -0.08227 \\
\hline & S5 & 2.63611 & 2.46194 & -0.07075 \\
\hline & P3 & 2.80837 & 2.98877 & 0.06036 \\
\hline & $\mathrm{P} 4$ & 2.75173 & 2.75688 & 0.00187 \\
\hline \multicolumn{5}{|c|}{$\begin{array}{l}\Delta m_{p}-\text { average weight change after } 5 \text { years of exposure calculated } \\
\text { of exposure } \\
\Delta m_{e}-\text { actual weight change determined after } 5 \text { years of exposure } \\
\Delta m=\left(\Delta m_{e}-\Delta m_{0}\right) / \Delta m_{e}-\text { relative model error }\end{array}$} \\
\hline
\end{tabular}

The regression curves fitted to the average weight changes of mortar samples modified with S4 glycolysates, after 3.5 years and 5 years of immersion in a $10 \%$ solution of sulphuric acid are shown in Figure 2 (logarithmic model), and Figure 3 (exponential model). The relative error for both models is comparable; however, the severalyear testing of samples immersed in other aggressive media shows that the logarithmic model is not always correct, and the exponential model performs better.

The exponential model used to describe the average weight changes of epoxy mortar samples immersed in a solution of sulphuric acid (Figure 3) suggests that the changes to this prop- erty will be larger, while actually they are a little smaller. This is a positive characteristic, in particular at the stage of designing products with such composites. Application of polymer composites in construction requires a certain reserve in the strength values to fit the expected level of durability, reliability and operational safety. This is especially important in the equipment that is subject to higher standards of required reliability and operational safety. This category of applications includes, inter alia, chemical resistant industrial equipment and building construction [Bełzowski 2004].

Figure 4 presents the logarithmic trend lines showing the average weight changes 
Table 4. Summary of expected and actual average mortar sample weight changes after 5 years of exposure in aggressive media, according to the exponential model

\begin{tabular}{|c|c|c|c|c|}
\hline Type of corrosive solution & Type of glycolysate & $\Delta m_{p}, \%$ & $\Delta \mathrm{m}_{\mathrm{e}}, \%$ & $\Delta \mathrm{m}$ \\
\hline \multirow{4}{*}{$\mathrm{H}_{2} \mathrm{SO}_{4}$} & S4 & 10.81706 & 8.85590 & -0.22145 \\
\hline & S5 & 9.97268 & 7.93176 & -0.25731 \\
\hline & P3 & 10.44979 & 8.47583 & -0.23289 \\
\hline & P4 & 9.89821 & 7.96731 & -0.24235 \\
\hline \multirow{4}{*}{$\mathrm{HNO}_{3}$} & S4 & 12.24876 & 9.97358 & -0.22812 \\
\hline & S5 & 10.86434 & 8.82926 & -0.23049 \\
\hline & P3 & 11.38432 & 9.36220 & -0.21599 \\
\hline & $\mathrm{P} 4$ & 11.24759 & 9.74383 & -0.15433 \\
\hline \multirow{4}{*}{$\mathrm{NaOH}$} & S4 & 3.28732 & 2.61884 & -0.25526 \\
\hline & S5 & 3.15656 & 2.46194 & -0.28214 \\
\hline & P3 & 3.38177 & 2.98877 & -0.13149 \\
\hline & $\mathrm{P} 4$ & 3.18019 & 2.75688 & -0.15355 \\
\hline \multicolumn{5}{|c|}{$\begin{array}{l}\Delta \mathrm{m}_{\mathrm{p}}-\text { average weight change after } 5 \text { years of exposure calculated } \\
\text { of exposure } \\
\Delta \mathrm{m}_{\mathrm{e}}-\text { actual weight change determined after } 5 \text { years of exposure } \\
\Delta \mathrm{m}=\left(\Delta \mathrm{m}_{\mathrm{e}}-\Delta \mathrm{m}_{\mathrm{e}}\right) / \Delta \mathrm{m}_{\mathrm{e}}-\text { relative model error }\end{array}$} \\
\hline
\end{tabular}

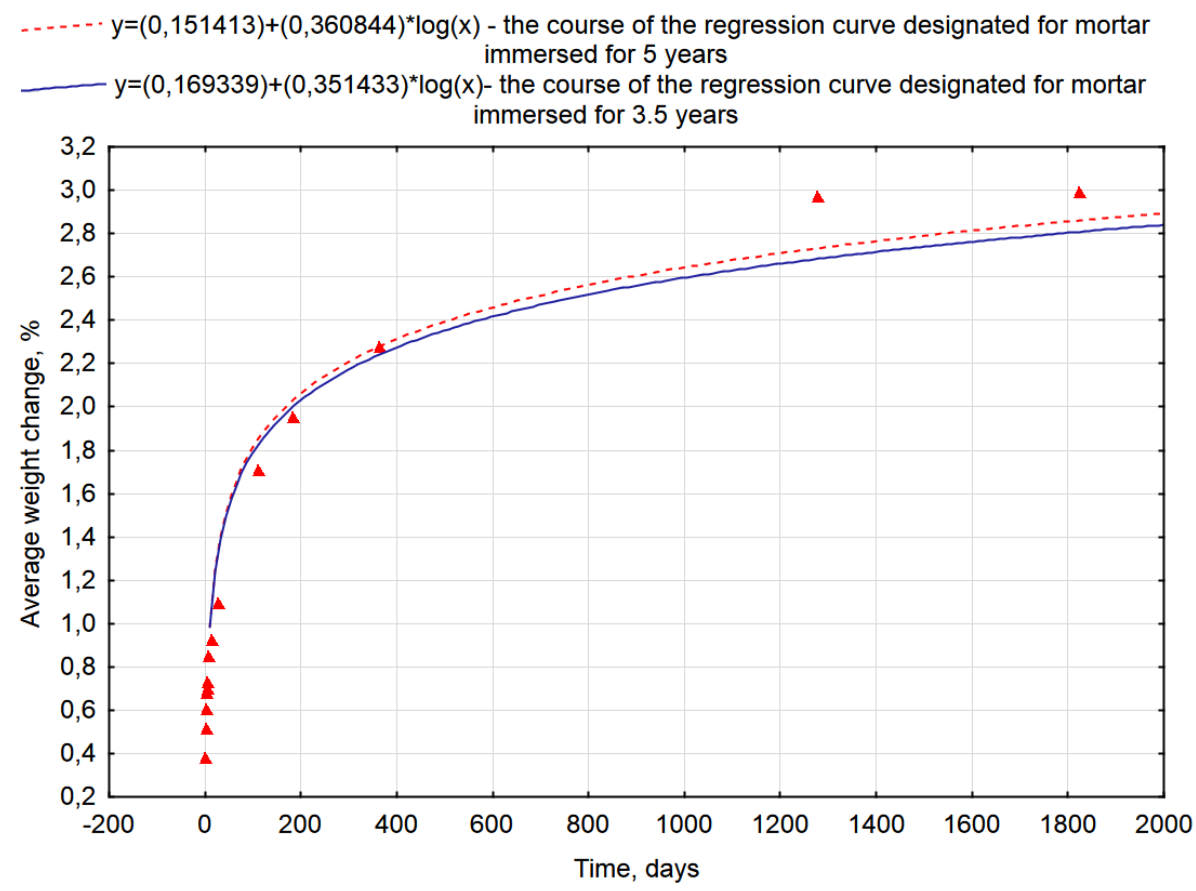

Figure 1. The course of the regression curve designated for mortar modified with P3 glycolysates, immersed for 3.5 years and 5 years in a $10 \%$ solution of $\mathrm{NaOH}$

observed during 5 years of testing for epoxy mortar samples modified with S4 glycolysates, immersed in four different corrosive media.

The least aggressive proved to be the sodium chloride sodium hydroxide solutions. The average sample weight changes after 5 years of exposure were c. $2 \%$ and $3 \%$. Regression curves for these solutions reached a plateau. The average weight changes of the samples immersed in aqueous solutions of sulphuric and nitric acids are much higher, at the level of $9-10 \%$, but the chemical resistance of epoxy mortar is still much better than ordinary cement mortar.

The long-term effect of solutions of acids on mortar samples led to the weakening of the connection between the aggregate and the matrix polymer, which resulted in the amount of solution absorbed. By observing the fractures in the mortar samples kept in sodium hydroxide solu- 


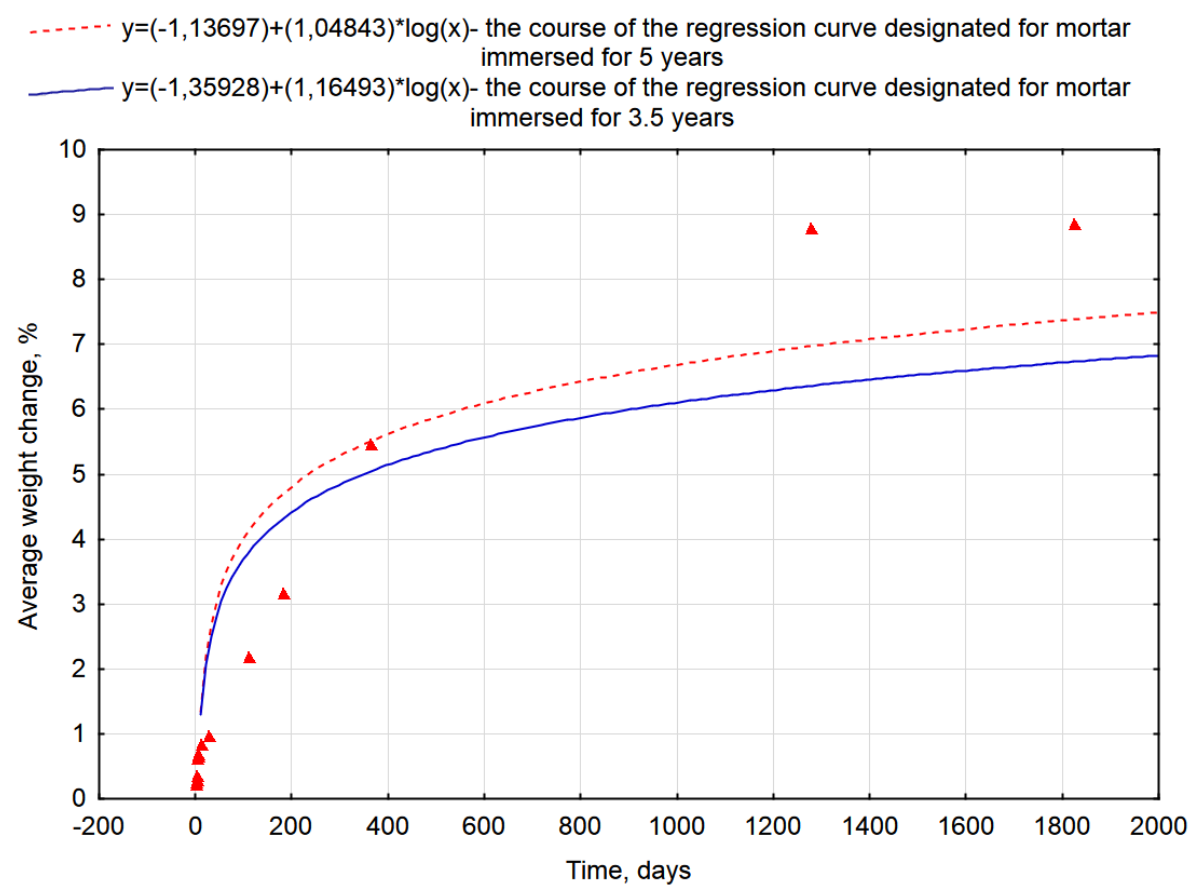

Figure 2. Regression curve fitted to average weight changes of mortar samples modified with S4 glycolysates, after 3.5 years and 5 years of immersion in $\mathrm{H}_{2} \mathrm{SO}_{4}$, logarithmic model

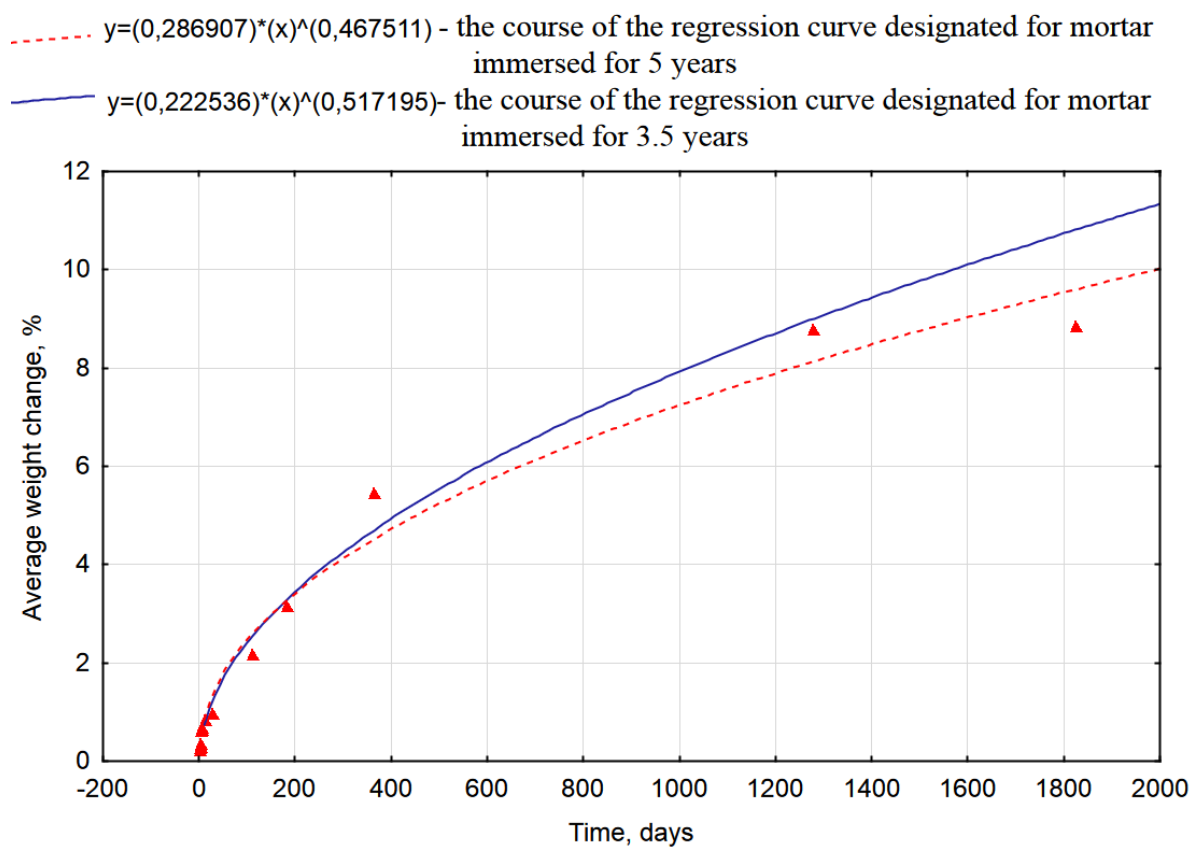

Figure 3. Regression curve fitted to average weight changes of mortar samples modified with S4 glycolysates, after 3.5 years and 5 years of immersion in $\mathrm{H}_{2} \mathrm{SO}_{4}$, exponential model

tion, it can be concluded that the resin is still well attached to the aggregate. After five years of exposure of samples in aqueous solutions of acids, they can easily be broken up by hand (Figure 5), which is not possible in the case of the samples immersed in aqueous solutions of sodium hydroxide and sodium chloride.

\section{CONCLUSIONS}

Carrying out long term studies on the chemical resistance of epoxy mortar modified with poly(ethylene terephthalate) glycolysates from waste allowed the formulation of the following conclusions: 


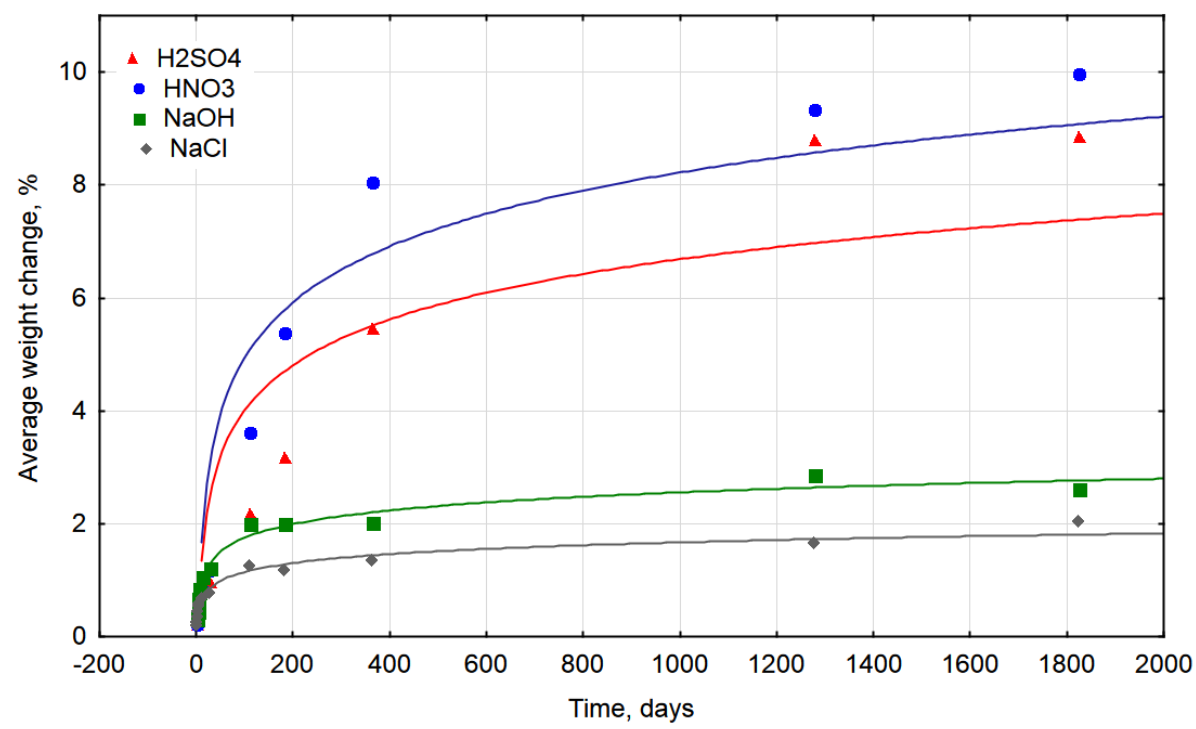

Figure 4. Logarithmic trend lines showing the average weight changes during 5 years in samples of epoxy mortar modified with S4 glycolysates, immersed in four different corrosive media

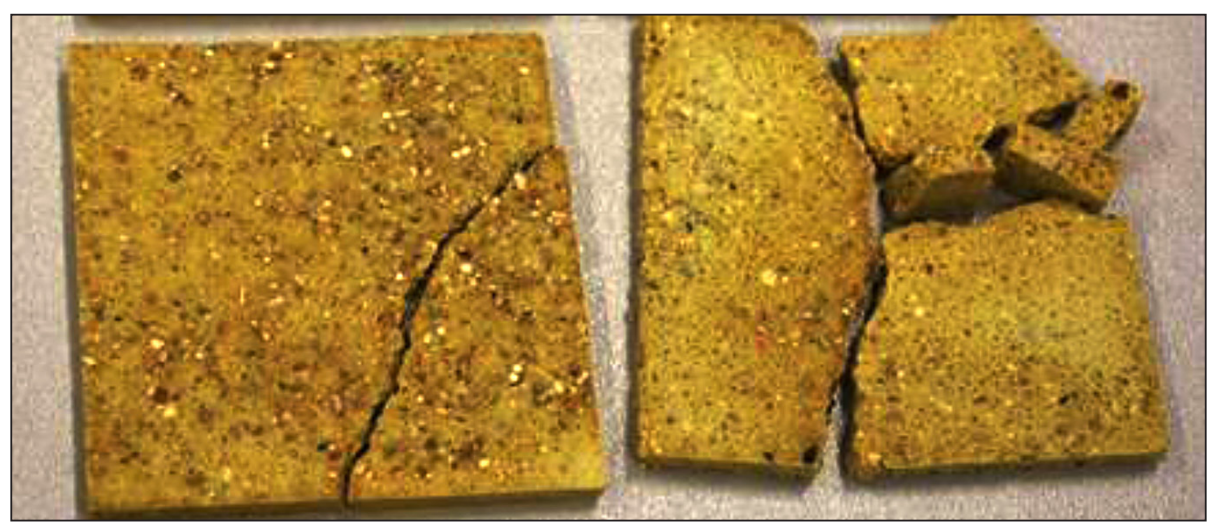

Figure 5. Samples after 5 years of exposure in $10 \%$ solution of nitric acid, broken up by hand

1. The average weight changes of modified epoxy mortar samples immersed in $10 \%$ aqueous solution of sodium chloride and sodium hydroxide over time begin to stabilize on a constant basis, c. $2 \%$ and $3 \%$, respectively. The logarithmic model describes the behavior of mortar samples immersed in these media well.

2. The average weight changes of modified epoxy mortar samples exposed to $10 \%$ aqueous solutions of sulphuric and nitric acids, in the period from 3.5 to 5 years from immersion further increased slightly to the values of, c. $9 \%$ and $10 \%$, respectively. The values measured after 5 years of exposure are different than expected on the basis of the trend line fitted to the data after 3.5 years of immersion.

3. The exponential model used to describe the average weight changes of epoxy mortar samples immersed in a solution of sulphuric acid suggests that the changes to this property will be larger, while in reality they are a little smaller. This approach seems to be safer for the design process, and therefore it was considered that the exponential curve models the tested phenomenon better.

4. In the samples of mortars immersed in aqueous solutions of acids, over time, the connection between the polymer matrix and aggregate weakens, which results in an increase in their weight and increasing fragility. After 5 years of exposure of the samples they can be very easily broken up.

5. Even after 5 years of immersion of modified epoxy mortar samples in aggressive media, their resistance is still greater than the resistance of cement mortar. 


\section{Acknowledgements}

The research work has been carried out with the use of funds granted by the Ministry of Science and Higher Education in the framework of the research promotion project 1943/B/T02/2010/38.

During the execution of the work, funds were also used from the European Social Fund and the State Budget and the Budget of the Podkarpackie Province of the Republic of Poland.

\section{REFERENCES}

1. Amaro A.M., Reis P.N.B., Neto M.A., Louro C. 2013. Effects of alkaline and acid solutions on glass/epoxy composites. Polymer Degradation Stability, 98, 853-862.

2. Bełzowski A. 2004. Rules for the selection of structural safety factors from composite materials. Composites, 4, 396-403 (in Polish).

3. Czarnecki L. 2010. Polymer Concretes. Cement Lime Concrete, 2, 63-85.

4. Czarnecki L., Sokołowska J.J. 2015. Material model and revealing the truth. Bulletin of the Polish Academy of Sciences. Technical Sciences, 63, 7-14.

5. Dębska B., Lichołai L. 2016. The effect of the type of curing agent on selected properties of epoxy mortar modified with PET glycolisate. Construction and Building Materials, 124, 11-19.

6. Feng P., Wang J., Wang Y., Loughery D., Niu D. 2014. Effects of corrosive environments on properties of pultruded GFRP plates. Composite Part B., 67, 427-433.

7. Garbacz A., Sokołowska J.J. 2013. Concrete-like polymer composites with fly ashes - Comparative study. Construction and Building Materials, 38, 689-699.

8. Garcia-Espinel J.D., Castro-Fresno D., Parbole Gayo P., Ballester-Muñoz F. 2015. Effects of sea water environment on glass fiber reinforced plastic materials used for marine civil engineering constructions. Materials Design, 66, 46-50.

9. Ghorbel E., Haidar M., 2016. Durability to Chemical Attack by Acids of Epoxy Microconcretes by Comparison to Cementitious Ones. Advances of Civil Engineering, 1-15.

10. Gizelter R. 2014. Buildings Materials and Structures Based on Advanced Polymer Nanostructured Matrix. International Letters of Chemistry, Physics and Astronomy, 9, 103-114.

11. Golestaneh M., Najafpour G., Amini G., Beygi M. 2013. Evaluation of Chemical Resistance of Polymer Concrete in Corrosive Environments. Iranica Journal of Energy and Environment 4 \{(3) Geohazards and Civil Engineering) $\}, 304-310$.
12. Gorninski J.P., Dal Molin D.C., Kazmierczak C.S. 2007. Strength degradation of polymer concrete in acidic environments. Cement and Concrete Composites, 29, 637-645.

13. Griffiths R., Ball A. 2000. An assessment of the properties and degradation behaviour of glassfibre-reinforced polyester polymer concrete. Composites Sciences Technology, 60, 2747-2753.

14. Lichołai L., Dębska B. 2014. The multidimensional response function exemplified by epoxy mortars: looking for the global extreme. Archives of Civil and Mechanical Engineering, 14, 466-475.

15. Lokuge W., Aravinthan T. 2013. Effect of fly ash on the behaviour of polymer concrete with different types of resin. Materials Design, 51, 175-181.

16. Lu T., Solis-Ramos E., Yi Y-B., Kumosa M. 2016. Synergistic environmental degradation of glass reinforced polymer composites. Polymer Degradation Stability, 131, 1-8.

17. Mortas N., Erb O., Reis P.N.B., Ferreira J.A.M. 2014. Effect of corrosive solutions on composites laminates subjected to low velocity impact loading. Composite Structures, 108, 205-211.

18. Rahimi R.R., Iman M.N., HamedA., Samira H.T. 2016. Sustainable approach for recycling waste tire rubber and polyethylene terephthalate (PET) to produce green concrete with resistance against sulfuric acid attack. Journal of Cleaner Production, 126, 166-177.

19. Rahman Md. M., Akhtarul Islam Md., Tamez Uddin Md. 2015. Excellent durability of epoxy modified mortars in corrosive environments. Journal of Polymer Engineering, 36, 79-85.

20. Reis J.M.L. 2010. Fracture assessment of polimer concrete in chemical degradation solutions. Construction and Building Materials, 24, 1708-1712.

21. Reis J.M.L. 2009. Mechanical characterization of polymer mortars exposed to degradation solutions. Construction and Building Materials, 23, 3328-3331.

22. Ribeiro M.C.S., Tavares C.M.L., Ferreira A.J.M. 2002. Chemical resistance of epoxy and polyester polymer concrete to acids and salts. Journal of Polymer Engineering, 22, 27-44.

23. Stamenović M., Putić S., Rakin M., Medjo B., Čikara D. 2011. Effect of alkaline and acidic solutions on the tensile properties of glass-polyester pipes. Materials Design, 32, 2456-2461.

24. Suihkonen R., Lindgren M., Siljander S., Sarlin E., Vuorinen J. 2016. Erosion wear of vinylester matrix composites in aqueous and acidic environments at elevated temperatures. Wear, 358-359, 7-16.

25. Yan L., Chouw N., Jayaraman K. 2015. Effect of UV and water spraying on the mechanical properties of flax fabric reinforced polymer composites used for civil engineering applications. Materials Design, 71, 17-25. 